\title{
INMIGRANTES EN ANDALUCÍA: ALGUNAS CLAVES PARA SU ESTUDIO DESDE LA ANTROPOLOGÍA CULTURAL
}

\author{
Emma MARTÍN DIAZ
}

\section{INTRODUCCIÓN: LA IMPORTANCIA DEL ESTUDIO DE LOS PROCESOS MIGRATORIOS EN LA ANDALUCÍA ACTUAL}

La primera reflexión que debemos hacernos es el gran desconocimiento que rodea a un tema de tanta trascendencia como es el de la presencia de colectivos de emigrantes mayoritariamente magrebíes y en menor medida centroafricanos en el territorio andaluz. La ausencia de estudios cuantitativos que sean representativos de la realidad numérica total de emigrantes en Andalucía, de su incidencia en los diversos sectores productivos, su nivel educativo, procedencia, y otros datos significativos es palmaria. En parte, esta ausencia estaría justificada por la que es una de las principales características de la actual inmigración: LA ILEGALIDAD, que acompaña y permite la inserción de los inmigrantes en lo que conocemos como economía sumergida. En este aspecto, es indudable que llevamos un importante retraso con respecto a otras comunidades autónomas como Cataluña o Madrid, donde sí existen estudios significativos aunque ello también puede deberse a que la inmigración en Andalucía es un fenómeno cronológicamente más reciente que en las dos comunidades citadas.

Si la ausencia de estudios cuantitativos es significativamente importante, aún más lo es la ausencia de estudios cualitativos que nos indiquen quiénes son estos inmigrantes: de qué tipo de realidad proceden, qué formas de organización social les son propias, qué mecanismos utilizan para dirigirse a los distintos puntos de destino, de qué forma se insertan en la sociedad receptora. Cuestiones que deben ser conocidas para determinar con éxito qué medidas son eficaces y cuál es el procedimiento adecuado para facilitar la convivencia y la inserción el el tejido social andaluz de los distintos colectivos de inmigrantes.

* Profesora titular de la Universidad de Sevilla. 
El desconocimiento al que hemos aludido es importante y grave, pero dentro del fenómeno migratorio hay otras realidades tan importantes como ésta que pocas veces son tenidas en cuenta. Una es la de los países de origen y los factores desencadenantes de la emigración. La otra es la propia realidad de los países receptores. No deja de ser lamentable el hecho de que una gran parte de los trabajos que se ocupan del fenómeno migratorio no tengan en cuenta ni la propia sociedad receptora ni la variabilidad de situaciones que se produce dentro de cualquier inmigración.

Antes de comenzar con el análisis de la estructura socioeconómica andaluza tenemos que señalar que en nuestro territorio coexisten dos tipos de inmigración bien diferentes: una inmigración laboral y lo que conoce como la "emigración en busca del sol". Esta última presenta varias características diferenciadoras: está compuesta por individuos provenientes de las capas altas y medias de los países más desarrollados del norte y el centro de Europa que, una vez acabada su etapa laboral, emigran en busca de un clima más cálido que el de sus países de origen. Estos individuos comienzan a instalarse en poblaciones de la costa andaluza durante los años sesenta, concentrándose fundamentalmente en la provincia de Málaga y aprovechando una coyuntura económica que les era altamente beneficiosa. Hoy, forman auténticas "colonias" de extranjeros sin apenas relación con los habitantes de las localidades. Sin embargo, las nuevas normas de la Comunidad Europea sobre el voto municipal van a influir de manera decisiva en el peso político de estos colectivos de emigrantes, peso que ya es considerable en algunas localidades. Pese a su importancia, nosotros nos vamos a centrar en la inmigración laboral, la cual no puede ser entendida sin hacer referencia a las transformaciones que han tenido lugar en Andalucía en los últimos treinta años, en particular en el sector agrícola.

\subsection{Los procesos migratorios y el sector agrícola andaluz}

Es una realidad que Andalucía ha experimentado importantes transformaciones cuyo origen hay que buscar en los años sesenta. Este proceso ha sido magníficamente analizado por M. Delgado y otros. $(1981,1985)$ En síntesis, podemos señalar que a comienzos de los años sesenta Andalucía estaba sujeta a un importante cambio de orientación económica, fruto de la política desarrollista seguida por el Estado. Si hasta ese momento había actuado como suministradora de materia prima y como financiadora del desarrollo industrial de otras zonas, a partir de estos años comienza a recibir inyecciones de capital tendentes a desarrollar en su intetior un mercado de consumidores, a recibir las industrias más contaminantes y peligrosas y a tecnificar su agricultura. Este proceso de "modemización" supone, en cuanto que conlleva la mecanización de los procesos de trabajo agrícolas que 
eran la fuente principal de oferta de trabajo en el medio rural andaluz, el paro estructural de una gran cantidad de jornaleros y la crisis de las pequeñas explotaciones. En esta coyuntura, la emigración, potenciada por el propio Estado, se convierte en la alternativa más viable para los jornaleros "sobrantes" y los campesinos arruinados. En los años sesenta y comienzo de los setenta, aproximadamente dos millones de andaluces se ven forzados a emigrar, integrándose mayoritariamente en lo que Portes (1985) denomina mercado de trabajo secundario. Esta emigración, aunque supuso el despoblamiento de las zonas más marginales de Andalucía, particularmente de las montañosas, no tuvo grandes repercusiones en las zonas llanas en el plano demográfico. Sin embargo, sí que contribuyó, al menos de manera tan eficaz como la represión, a evitar las tensiones sociales que hubieran producido tales transformaciones.

De manera que puede parecer sorprendente, a los veinte años de comenzar un proceso como la emigración, que marcó considerablemente no sólo al territorio, sino a la propia etnicidad andaluza, (Moreno, 1981, 1986) Andalucía comienza a convertirse en receptora de una inmigración procedente del Magreb y de Africa Central, con un predominio de marroquíes, que se instalan preferentemente en el mismo sector del que salieron mayoritariamente los emigrantes andaluces: el agrícola. Esta inmigración coexiste con unos altísimos índices de desempleo en el sector agrícola.

El fenómeno, aunque reciente cronológicamente hablando, no es exclusivo de Andalucía, y sólo puede ser entendido en el contexto de los nuevos modelos adoptados en la división internacional del trabajo. En las actuales circunstancias de endurecimiento de las condiciones de vida para las capas más pobres de la población mundial, son los países dependientes, los más densamente poblados a su vez, quienes sufren en mayor medida el deterioro de la situación. La presión demográfica, la fuerte diferenciación salarial entre países desarrollados y dependientes, el conocimiento y difusión, política e ideológicamente interesadas, de los modos de vida occidentales, son fenómenos que explican la recurrencia masiva a la emigración, (Khader, 1992). Junto a estos motivos, la razón del auge migratorio actual hay que buscarla en la demanda efectiva de fuerza de trabajo inmigrante existente en determinados países y sectores económicos en los que se insertaba la emigración hacia Europa durante los años sesenta.

Estas características diferenciadoras son las que han llevado a algunos economistas y sociólogos italianos a denominar a la actual inmigración como "nueva inmigración". (Mottura y Pugliese, 1992) Para estos autores, la nueva inmigración presenta unas importantes características diferenciales: en primer lugar, tiene como puntos de destino países y regiones que han sufrido una importante emigración en años recientes: básicamente el norte mediterráneo. En segundo lugar, es una inmigración que tiene como destino primordial el sector agrícola, invirtiendo la dirección rural-urbana, agrícola-industrial, del modelo clásico. Por otra parte, esta in- 
migración se inserta en un mercado de trabajo autóctono que presenta unos fuertes índices de población activa desempleada, en un contexto de creciente feminización e incorporación de los más jóvenes, en sustitución de los cabezas de familia que tradicionalmente ocupaban este sector. (Pugliese, 1992) En este contexto, la inmigración actual o "nueva inmigración" se produce en una coyuntura de fuerte restricción de la inmigración y en un clima de creciente rechazo por parte de la población autóctona, rechazo unido a actitudes racistas y xenófobas.

En el caso andaluz, las razones que explican esta inmigración requieren de un análisis de la realidad social actual. Una de ellas es la existencia de una amplia población rural subsidiada, que suele complementar sus ingresos con una pluriactividad exonómica. Los costes crecientes de alojamiento y manutención y el aumento del nivel de vida de los jornaleros andaluces hace a éstos poco rentable el trasladarse de campaña en campaña. Junto a ello, tenemos que señalar la existencia de unos empresarios agrícolas de tipo familiar totalmente impotentes ante una política comunitaria tendente al mantenimiento e incluso el descenso de los precios agrícolas, que favorece la importación de productos agrícolas más baratos provenientes de países extracomunitarios. La, en muchos casos, única estrategia para rentabilizar las explotaciones familiares es el recurso a la sobreexplotación de la fuerza de trabajo ajena. En este contexto, es evidente que los trabajadores ilegales son mucho más fáciles de sobreexplotar. De esta forma, si no se pueden incrementar los precios del producto, se consigue abaratar los costes de producción y mantener, o al menos paliar en lo posible, la pérdida de beneficios.

En esta situación, y pese a la importante presión institucional por acabar con la inmigración por parte de la Comunidad Europea, ésta se seguirá produciendo porque es económicamente rentable. En un primer momento los inmigrantes se insertaron básicamente en la "nueva agricultura" debido a la amplia demanda de fuerza de trabajo de algunos cultivos y a las propias características de esta fuerza de trabajo inmigrada, que tiene que aceptar las duras condiciones de explotación existente. Pero, cada vez más, podemos encontrar también a inmigrantes compitiendo con jornaleros en áreas de fuerte predominio de este colectivo, con los problemas que esta situación de competencia por puestos de trabajo escasos puede llegar a plantear.

Los otros sectores de actividad en los que se insertan los inmigrantes son la venta ambulante y el servicio doméstico, aunque hay un número poco significativo de profesionales y técnicos y de pequeños y medianos empresarios.

\section{2. ¿Andalucía: país de tránsito o país de destino?}

Una de las características fundamentales de los fenómenos migratorios actuales es la dependencia de las coyunturas internacionales. Por tanto, resulta extre- 
madamente difícil afirmar si los países de destino son países de establecimiento definitivo o temporal de los inmigrantes. Como he podido comprobar en mis trabajos anteriores (Martín, 1985, 1992) resulta muy difícil señalar "a priori" si la emigración va a ser transitoria o estable, ya que ello depende de varias causas entre las cuales la voluntad de los emigrantes es dependiente de otras circunstancias que resultan del desarrollo de su propia experiencia migratoria y de la actuación política de los países receptores con respecto a la inmigración. En el contexto de la división internacional del trabajo que está en la base de los procesos migratorios actuales, los emigrantes son una mercancía más y su capacidad de toma de decisiones es muy pequeña. Esta dependencia y precariedad de situaciones hace que sea necesario tener en cuenta las distintas coyunturas a la hora de definir el tipo de inserción social -provisional o estable- de la inmigración en los diferentes lugares de destino.

En lo que se refiere a Andalucía, la repercusión de la inmigración empieza a notarse a partir de los años ochenta, cuando en lugares como Madrid o Cataluña existe ya un asentamiento importante de diferentes colectivos de inmigrantes. Africa es el lugar de procedencia de la mayoría de ellos debido a la proximidad geográfica de nuestras costas y las del continente africano. Andalucía se convierte en el lugar de recepción de la inmigración africana en un coyuntura de difícil permeabilidad de las fronteras de los países europeos receptores tradicionales de inmigración. En un primer momento, esta situación se ve favorecida por la escasa definición de la política española con respecto a la inmigración, ya que esta había sido prácticamente inexistente. La situación comienza a cambiar a partir de 1985 con la promulación de la Ley Orgánica sobre Derechos y Libertades de los Extranjeros. Muchos de los emigrantes que habían entrado hasta entonces lo habían hecho como turistas, un $64 \%$ según la encuesta del colectivo IOE.

El hecho de la permeabilidad de nuestras fronteras no explica la permanencia de la inmigración. Si en un primer momento la idea de los inmigrantes era dirigirse a los países desarrollados de la Europa Occidental, su establecimiento en el Estado español se debe más a las posibilidades de encontrar aquí trabajo que a la pretendida imposibilidad de encontrarlo en otros lugares. De igual forma, si Andalucía comenzó siendo un lugar de tránsito, es ahora un lugar de destino debido fundamentalmente a las características del mercado de trabajo rural andaluz a las que nos hemos referido. Una vez establecidos los primeros inmigrantes en este sector productivo, las redes sociales empiezan a funcionar e irán llegando nuevos inmigrantes que se refugiarán en diferentes sectores según las distintas provincias y las actividades económicas predominantes en las mismas. Paradójicamente, los datos sobre la inmigración legal parecen contradecir esta realidad. Según Del Moral (1993), Málaga es la rpovincia con un mayor número de inmigrantes, unos 10.000, empleados mayoritariamente en el sector terciario -básicamente en el turismo-. La mayoría de los 6.000 inmigrantes residentes en Almería trabajan en la agricultura. 
La construcción y el sector servicios acogen a la pràctica totalidad de los inmigrantes que residen en Sevilla, mientras que Huelva, debido a la existencia de empresas pesqueras mixtas hispano-maroquíes, cuenta con un alto índice de pescadores entre los 1.400 emigrantes estimados. Las razones que pueden explicar que el número de inmigrantes legales que se dedican a la agricultura sea tan bajo hay que buscarlas en la recurrencia, dentro de esta actividad económica, a la mano de obra ilegal, más fácilmente explotable y con menos costes para el empresario, al no tener que incluir en el REA a estos trabajadores.

El objetivo de nuestro estudio es contribuir a un mayor conocimiento de la inmigración en Andalucía basándonos en el análisis de los tipos de inserción social de los inmigrantes, de las redes sociales establecidas tanto en sus respectivos países como en los lugares de destino actual, de su percepción de los entornos de origen y de acogida, de sus expectativas, así como en el estudio de los modos concretos que adquieren las relaciones interétnicas y las actitudes y valores de los autóctonos con respecto a los diversos tipos de emigrantes.

Consideramos que un estudio de estas características resulta no sólo pertinenete, sino necesario, ya que si en algo coinciden los todavía escasos y dispares análisis realizados sobre la inmigración en España es en el hecho de que, pese a todas las trabas y cortapisas oficiales, ésta se seguirá produciendo. Así, aunque trabajos como los del colectivo IOE (1986) y los de A. Izquierdo (1990) planteen disparidades acerca del número real de inmigrantes en el territorio español, coinciden en señalar que estamos en los inicios de un fenómeno que va a continuar en los años venideros.

La mayoría de los expertos coinciden en afirmar que la crisis económica por la que estamos atravesando no va a incidir negativamente en el flujo inmigratorio. En lo que se refiere a los países de destino, porque los sectores sumergidos de la economía que dan empleo a la inmigración distan mucho de desaparecer. Por lo que respecta a los países de origen, porque la crisis va a agudizar todavía más las ya extremas condiciones de vida que se dan en los mismos.

En el caso del Mediterráneo, los fuertes desequilibrios entre el norte y el sur han sido el factor desencadenante de la inmigración proveniente de los países del Magreb, y una de las consecuencias de una relación tan problemática como intensa entre ambas orillas. Estamos de acuerdo con el profesor Khader (1992: 32) cuando señala que "en un contexto semejante, no existe ningún control de policía, ningún "cordón sanitario" que pueda contener los nuevos flujos migratorios en el Mediterráneo. Si no se alcanza un desarrollo económico en la ribera sur que disuada a los emigrantes en potencia, que los retenga en sus países, que les quite las ganas de marchar, de huir, vamos a asistir en las próximas décadas a una intensificación de los movimientos migratorios internos y de los flujos migratorios hacia el exterior". En este contexto, un adecuado conocimiento de la realidad se convierte en imprescindible. 


\section{LA ANTROPOLOGÍA Y EL ESTUDIO DE LOS PROCESOS MIGRATORIOS}

La relación de la antropología con el estudio de las migraciones es tan estrecha como fecunda. Dedicada en un primer momento al estudio de las sociedades lejanas y exóticas, los antropólogos no pudieron dejar de pasar por alto el fenómeno cultural que supone el encuentro con el "otro" en las sociedades propias. La literatura es abundante y va desde el análisis de las motivaciones individuales y del impacto de la modernización hasta los trabajos que se centran en el análisis de los mecanismos que desencadenan la inmigración, pasando por el análisis de las sociedades y grupos sociales concretos inmersos en el proceso. En una recensión, conviene destacar el análisis del papel de instituciones como el parentesco (Meillassoux, 1960), el rol de las redes sociales y familiares de los inmigrantes (Katuszewski y Ogien, 1981) el análisis de la interacción entre factores como la etnicidad y la clase social, (Epstein, 1969) así como el estudio del mercado de trabajo (Portes, 1985), y del papel de los estados (Walton, 1985) por citar sólo algunos de los puntos claves tratados por y desde la Antropología, y que han contribuído eficazmente a un mayor conocimiento y comprensión de los mecanismos inherentes a los procesos migratorios.

Desde las perspectivas situacional y procesual, los antropólogos han dado interesantes claves para comprender el fenómeno migratorio. Mi planteamiento es que debemos considerar a la emigración como un proceso. De esta forma tendremos presente su dimensión histórica y el hecho de que la emigración no se cierra cuando el emigrante llega a la sociedad de destino, que se continúa con la experiencia de retorno. La emigración es un proceso que afecta a las sociedades emisoras y receptoras, y no sólo en los planos demográfico y económico, sino también en el ámbito social, político e ideológico, y los antropólogos, como científicos sociales dedicados al estudio de los fenómenos culturales, se encuentran en una posición privilegiada para acceder a un adecuado conocimiento de este fenómeno.

\section{APUNTES METODOLOGICOS PARA EL ESTUDIO DE LA INMIGRACIÓN EN ANDALUCÍA}

Dentro de estos planteamientos teórico-metodológicos, un estudio de la inmigración en Andalucía debe tener en cuenta los distintos niveles y las diferentes dimensiones del fenómeno. Analizar qué supone la inmigración en Andalucía implica estudia tanto los distintos colectivos de inmigrantes como la realidad de los lugares de destino, las formas y mecanismos de inserción social, los resultados que se derivan de los procesos migratorios y la percepción que tienen del fenómeno los diferentes colectivos de autóctonos e inmigrados puestos en contacto mediante este proceso. 
En primer lugar, se hace necesaria una caracterización de los diferentes colectivos de inmigrantes existentes en Andalucía. Señalar que los principales motivos para emigrar son las necesidades económicas es una obviedad que necesita ser desarrollada. O lo que es igual, hay que analizar no sólo la situación objetiva de los países de procedencia, sino la situación de partida de los emigrantes. Esto es importante para caracterizar el tipo de inmigración y las expectativas de los inmigrantes. Muchas veces se da por supuesto oque los individuos abandonan sus países de origen para establecerse en los países más desarrollados y con mayores oportunidades de empleo. Sin embargo, no son extrañas las ocasiones en las que el inmigrante es un enviado de su propio grupo doméstico con el objeto de obtener un dinero extra para rentabilizar sus explotaciones, como sucede con parte de la emigración mexicana a los EE.UU. (Palerm, 1992) Dentro de algunos colectivos de inmigrantes, la salida de los individuos más jóvenes supone también para éstos la posibilidad de romper con la férrea autoridad del cabeza de familia y establecerse por su cuenta, en un mundo en el que las transformaciones sociales y económicas suponen una descomposición de las estructuras familiares tradicionales (Meillassoux, 1960). Por otra parte, no es extraño que la emigración suponga un fuerte crecimiento y desarrollo de una serie de ciudades en los países de origen, en las que se establecen primordialmente aquellos que han pasado parte de su vida activa en la emigración, y que deciden invertir sus ahorros en lugares menos deprimidos que sus regiones de procedencia, como sucede con Guadalajara en México. (Gonzáles, Escobar y Roberts, 1987) La propia realidad de los emigrantes andaluces nos señala que debemos ser muy cuidadosos a la hora de generalizar sobre las causas de la emigración y los objetivos que persiguen los emigrantes, así como sobre los resultados de la emigración, los cuales están sujetos a la propia evolución económica y social de las sociedades emisoras y receptoras, que depende a su vez de las distintas coyuturas nacionales e internacionales. (Martín, 1985, 1992).

Igualmente, un conocimiento adecuado de estos factores es indispensable para cualquier planteamiento aplicado tendente a favorecer la inserción social de los inmigrantes, quienes tienen necesidades y expectativas específicas y diferentes.

Junto al análisis de los intereses y objetivos de los inmigrantes, hay que tener en cuenta el tipo de sociedad en la que se insertan. Por tanto, se hace necesario destacar la diferencia entre los procesos migratorios que se dan en otros territorios del Estado español, como Cataluña o Madrid, y los que se dan en Andalucía. Sólo de esta forma estaremos en condiciones de comenzar a desarrollar un análisis de lo que supone la inmigración para Andalicía.

Dentro de este contexto, la inmigración presenta una serie de repercusiones sobre la estructura económica y social de Andalucía que deben ser analizadas en sus diferentes niveles: 
- Un nivel sería el de las formas de inserción de los inmigrantes en la estructura económica andaluza, sectores de actividad y papel de estos colectivos dentro de los procesos productivos específicos. En este sentido, es evidente que la "nueva agricultura" constituye un sector crucial en el análisis de los procesos inmigratorios $\mathrm{El}$ análisis de las formas y mecanismos de contratación, del tipo de inserción en el proceso productivo, las relaciones con los trabajadores autóctonos que realizan el mismo trabajo, con los superiores y con los empresarios, el grado de conocimiento de los procesos de trabajo y las formas de aprendizaje, la visión que tienen del trabajo que desempeñan, el grado de relación con los sindicatos y las organizaciones existentes en su entorno laboral, es decir, todos los factores que conforman las "culturas del trabajo" presentes en estos procesos, forman una parte esencial de la investigación, que debe complementarse con el análisis de qué suponen los diferentes sectores económicos con presencia inmigrada en el conjunto de la economía andaluza y cual es la función de los inmigrantes en los mismos. Este análisis nos situará en condiciones de afirmar si la inmigración es, como señalan algunos investigadores, un fenómeno independiente de las necesidades de los sectores económicos de acogida, o si, por el contrario, tiene una clara funcionalidad dentro de los mismos.

- Junto a la inserción en la estructura económica habría que analizar las formas de inserción de los distintos colectivos inmigrantes en la sociedad andaluza y los problemas que éstas plantean y pueden plantear en un futuro próximo, ya que estamos hablando de un fenómeno que tiende a incrementarse de manera progresiva. Al igual que la inmigración no afecta por igual a todos los sectores económicos, tampoco afecta por igual al conjunto de la sociedad andaluza. Frente a la concentración en las grandes ciudades industriales, característica de las migraciones de los años sesenta, la "nueva inmigración" presenta una concentración importante de inmigrantes en aquellas localidades con un claro predominio de la agricultura intensiva. Aunque el número absoluto de inmigrantes que viven en estas localidades sea menor que el número de inmigrantes que viven en algunas capitales andaluzas, en ellas el porcentaje es mucho mayor y su peso específico bastante más considerable.

La inmigración en Andalucía se inserta en una estructura social que ha sufrido importantes modificaciones en lo que refiere a la composición de los sectores tradicionalmente presentes en el medio rural. Esta transformación es particularmente importante en las zonas costeras andaluzas, lugares de concentración de la inmigración y donde se ha propiciado la sustitución de las élites locales tradicionales por un sector de nuevos agricultores enriquecidos. (Martín y Melero, 1991, 1993) El análisis de la inserción de los inmigrantes en un contexto como éste, donde 
destaca el reforzamiento de una ideología "igualtarista", basada en la consideración de que "los señoritos ya no son lo que eran", debe tener en cuenta la posible cohesión de un "nosotros" local frente al "ellos" que suponen los inmigrantes. La existencia de roces o la posible separación de los inmigrantes de la vida social de las localidades es un punto central en nuestro análisis. Por otra parte, los roces pueden presentarse en localidades con un fuerte predominio jornalero, en las que comienzan a aparecer inmigrantes en los periodos de campañas agrícolas. En este caso, la competencia por el trabajo es un factor esencial.

El análisis de las repercusiones de la inmigración sobre la estructura social andaluza debe relacionarse con la articulación de ésta con la realidad estatal. La existencia de una política estatal de inmigración determina las acciones políticas emprendidas por la administración en sus diferentes niveles: central, autonómico y estatal. El análisis de las contradicciones entre una política restrictiva, tendente a limitar y legalizar la inmigración, y una práctica cotidiana tendente a potenciarla manteniendo la ilegalidad del fenómeno, es crucial para entender el fracaso de la actual política migratoria, aunque no sea la única razón. La situación económica y sociopolítica de los países emisores constituye el otro factor explicativo de este fracaso.

Dentro de la percepción simbólica de las repercusiones de la inmigración en la estructura andaluza destaca la contradicción existente entre la todavía no muy numerosa presencia de inmigrantes en Andalucía y la percepción de que éste es uno de los principales problemas de nuestro territorio. Las causas de esta contradicción sólo podrán ser puestas de relieve mediante un conocimiento exhaustivo de la realidad económica y social andaluza.

Coherentes con lo expuesto, consideramos que en un estudio sobre la inmigración se deben acotar los siguientes niveles de análisis.

- Los individuos y/o grupos domésticos inmigrantes y las estrategias económicas de los mismos.

- El/los sectores productivos con presencia inmigrada.

- Los diferentes colectivos de inmigrados, centrándonos en el análisis del colectivo marroquí y su interacción con los sectores sociales relevantes en el proceso de las relaciones interétnicas dentro de las localidades.

El individuo es el sujeto de la experiencia migratoria pero su importancia está claramente sobredimensionada en buena parte de los estudios sobre emigración. La mayoría de éstos se han centrado en el análisis de las motivaciones individuales que, según los partidarios de una antropología basada en la propia percepción de los protagonistas, están en la misma base y son la causa que explica la emigración. En la mayoría de estos estudios abundan las explicaciones causales de carácter psicologista, a veces amparadas en la teoría de la "modernización" como co- 
bertura teórica, para concluir señalando que es la desestructuración del mundo "rural", y la penetración de los "valores urbanos" el factor clave en la "decisión" de abandonar las "comunidades" de origen. En el fondo, y a pesar de los distintos grados de rigor, calidad y validez, estos trabajos son una variante de la teoría del push-pull, representados en sus polos por las dimensiones rural y urbana, dimensiones presentadas como universos distintos y contrapuestos.

Pese al abuso del análisis individual, éste resulta necesario aunque no es la única dimensión a tener en cuenta. No tanto porque, como se ha afirmado hasta la saciedad, la inmigración sea una decisión de carácter individual, sino porque, independientemente de los factores ajenos al individuo que pueden estar en la base de su decisión de abandonar el lugar de origen, ésta es una experiencia en la que el propio bagaje individual adquiere una importancia fundamental. La emigración no supone lo mismo para los individuos solteros que para los casados, para los campesinos que para los obreros, para los hijos mayores que para los más jóvenes, hay toda una variedad de situaciones que es preciso contemplar, y que condicionan la propia experiencia migratoria.

La variedad de situaciones no supone ningún obstáculo para la investigación. Nuestra experiencia nos señala que es bastante factible que existan las suficientes similitudes entre los individuos como para poder establecer una tipología de inmigrantes, que nos ayude a conocer cuáles son sus motivaciones y expectativas.

El estudio de los sectores productivos de inserción es otro nivel de análisis fundamental. En primer lugar, un conocimiento adecuado del funcionamiento y necesidades de estos sectores nos permitirá conocer la demanda real de fuerza de trabajo inmigrada. Pero lo que confiere a este nivel su dimensión fundamental es que son los procesos productivo, sus características y especificidades, los factores que explican la continuidad de la presencia -en unas condicones determinadas- de los inmigrantes en una zona concreta, asi como su forma específica de inserción social en la misma. Dentro de estos procesos se desarrollan la mayor parte de las relaciones entre autóctonos e inmigrantes, aún muy poco integrados en el resto del tejido social. Así, las relaciones que se establecen entre los inmigrantes y los autóctonos involucrados en un mismo proceso productivo (empresarios, asalariado y capataces), viene marcada por la existencia de intereses y objetivos distintos, y por distintas percepciones de los procesos de trabajo. El análisis de la existencia de unas culturas del trabajo específicas para los distintos individuos puestos en contacto en el interior de los procesos productivos constituye un elemento central de la investigación.

El análisis de los distintos colectivos que la inmigración pone en contacto debe ser uno de los puntos centrales de toda investigación sobre la inmigración, pero el problema es que no siempre se realiza. Gran parte de los estudios tienden a contraponer autóctonos e inmigrantes, presentándolos como dos bloque homogéneos y cohesionados, y limitándose a señalar las relaciones que se establecen entre es- 
tos dos presuntos bloques en términos de integración/asimilación, o de rechazo y xenofobia. La cuestión es bastante más compleja. En primer lugar, no es cierto que los inmigrantes sean un bloque compacto y homogéneo: como ellos mismos se encarga de afirmar, cada vez con mayor énfasis, pertenecen a grupos étnicos diferenciados y poseen, por tanto, una cultura específica que quieren conservar y defender. La creación en la capital granadina de una emisora específica de y para los senegaleses residentes en la zona es un ejemplo de cómo se intenta salvaguardar la cultura de origen. Pese a los evidentes problemas que el reconocimiento de la diversidad cultural plantea en una sociedad como la andaluza, poco acostumbrada al pluralismo étnico en los últimos quinientos años de su historia, salvo para el caso de los gitanos, se hace cada vez más necesario el trabajo de los científicos sociales en el campo de las relaciones interétnicas, y mas en un contexto como el actual, marcado por la extensión de conductas racistas y xenófobas.

\section{BIBLIOGRAFÍA CITADA}

AGUDO, J.; CRUCES, C. y MARTÍN, E. (1993): "Intensificación agraria y transformaciones sociales en Andalucía" Coloquio Internacional Andalucía y el Norte de Marruecos: Antecedentes históricos y situación actual. Sevilla, Marzo de 1993.

BALLARD, R. (1987): "The Political Economy of Migration: Pakistan, Britain and the Midle East" EADES, J. (ed.) Migrants, Workers and the Social Order, Tavistock Publications, London, pp. 17-41

COLECTIVO IOE (1992): Inmigrantes en España. Marco interpretativo de las principales tendencias. Fundación Friedrich Ebert. Intituto Fe y Secularidad, Madrid (en prensa)

DELGADO, M. (1981): Dependencia y marginación de la economía andaluza. Publicaciones del Monte de Piedad y Caja de Ahorros de Córdoba.

DELGADO, M. (1988, 1989): "Algunos rasgos de la economía andaluza en la crisis. 1973-1985", Revista de Estudios Andaluces, 10 y 11.

DELGADO, M. (1991): "La dinámica económica en Andalucía", en VV.AA. Interdependencia $e$ identidad andaluza ante la integración europea Ed. Centro de estudios Ramón Areces, S. A. Madrid.

DEL MORAL, J. M. (1993): "Inmigrantes en Andalucía”, en Carta de España. Revista de emigración e inmigración, 469

EADES, J. (1987): Migrants, Workers and the Social Order. London, Tavistock.

EPSTEIN, A.L. (1958): Politics in an Urban African Community. Manchester U.P.

IZQUIERDO, A. (1992): La inmigración en España. Ministerio de Trabajo y seguridad Social.

KHADER, B. (1992): Europa y el Gran Magreb, Fundación Paulino Torras Domènech, Barcelona.

MARTÍN, E. (1985): "Aspectos socioculturales de la emigración en la Sierra de Cádiz: el retorno", Cádiz y su provincia, vol. IV, pp. 123-147

MARTÍN, E. (1992): La emigración andaluza a Cataluña. Identidad cultural y papel político. Fundación Blas Infante, Sevilla. 
MARTÍN, E.; MELERO, M.L. (1992): "Intensificación de la agricultura y cambios en la estructura social y el sistema simbólico en la zona de Palos-Moguer (Huelva) Campaña 1989-90". Anuario de Etnología, Consejería de Cultura de la Junta de Andalucía, pp. 137-147.

MARTÍN, E.; MELERO, M.L. (1993): "Intensificación de la agricultura y cambios en la estructura social y el sistema simbólico en la zona de Palos y Moguer. Campaña de 1990-91". Anuario de Etnología, Consejería de Cultura de la Junta de Andalucía. pp. 21-28.

MEILLASSOUX, C. (1960): "Essai d'interpretation du phénoméne economique dans les societés traditionelles d'autosubsistence", Cahiers d'Etudes Africaines, 1 (4): 38-61.

MORENO, I. (1981): "Hacia la generalización de la conciencia de etnicidad (1936-1981)", Historia de Andalucía, (A. Domínguez Ortiz, Dir.), vol VIII, pp. 275-298.

MORENO, I. (1985): "Etnicidad, conciencia de etnicidad y movimientos nacionalistas: aproximación al caso andaluz", Revista de Estudios Andaluces, 5 pp. 13-83.

MORENO, I. (1986): "La identidad andaluza: pasado y presente (Una aproximación antropológica)". Andalucía (M. Barrera, ed.), pp. 253-284.

MOTTURA, G. (1992): "Forme della presenza extracomunitaria nell'agricoltura italiana: resultati di una prima esplorazione". Aspetti economici dell'inmigrazione in Italia.

PALERM, J.V. (1991): "Farm Labor Needs and Farm Workers in California: 1979 to 1989", California Agricutural Studies, 91 (2).

PORTES, A. (1981): "Modes of Structural Incorporation and Present Theories of Labor Migration”, KRITZ, KEELY \& TOMASI (eds.) Global Trends in Migration: Theory and Research on International Population Movements. Staten Island, N. Y. Center for Migration Studies of New York, Inc., pp. 279-297.

PUGLIESE, E. (1992): "L'inmigrazione in Italia: un confronto tra Nord e Sud". Ponencia presentada al curso de la UIMP Trabajadores inmigrantes en la agricultura mediterránea, dirigido por C. Giménez, Valencia, Septiembre de 1992. 